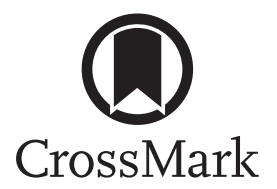

\title{
Progress in the management of IPF-related acute exacerbations: a goal for patients, respirologists and intensivists
}

\author{
To the Editor:
}

We read with interest the illuminating review on the pharmacological management of idiopathic pulmonary fibrosis (IPF) recently published in the European Respiratory Journal [1]. RAGHU [1] did not detail the pharmacological management of acute exacerbations (AEs). AEs often lead to the question of whether to transfer the patient to the intensive care unit (ICU) for respiratory support, whether this is invasive mechanical ventilation (IMV) or not, and to the choice of adequate pharmacological agent(s) [2]. This is a critical issue for respirologists and intensivists as it is estimated that $\sim 3$ million patients suffer from this disease worldwide [3]. When making the decision of whether to transfer to the ICU, it should be kept in mind that $\leqslant 46 \%$ of IPF-related deaths are preceded by an AE, with the majority of patients dying within the first month and most of the remaining within a year [2, 4].

The 2011 guidelines from the American Thoracic Society (ATS)/European Respiratory Society (ERS)/Japanese Respiratory Society (JRS)/Latin American Thoracic Association (ALAT) suggested that IMV might only be reasonable in a minority of patients (weak recommendation, low-quality evidence) [5]. This point was not discussed in the updates published in 2015 [6]. French experts published their recommendations in 2017 [7]. ICU management in a patient with IPF-related AEs appears to be justified when there is a pulmonary transplant, when a reversible cause of exacerbation has been identified, or if the research has not been carried out [7]. It should only be used after discussion with the patients and their relatives (ideally in advance).

The identification of patients who can best benefit from admission to the ICU remains a challenge. The Glasgow Prognostic Score was recently demonstrated to be advantageous in the classification of patients with IPF-related AEs into three disease stages with distinct mortality risk over 1-3 years [8]. Its usefulness in the decision to transfer the patient to the ICU requires validation in prospective cohorts performed in the era of new pharmacological treatments described by RaGHU [1].

The data on the symptomatic management of IPF-related AEs (i.e. respiratory support) were recently reviewed by KONDOH et al. [2]. Only four out of five studies published since 2008 included patients in the ICU (182 patients). Data on oxygenation with "new" techniques are lacking. Humidified high-flow nasal cannula oxygen could perhaps be used for long periods, possibly when waiting for lung transplantation [9]. Recent findings suggest that noninvasive ventilation failure could be deleterious as it increases the risk of ventilator-induced lung injury [10]. Extracorporeal oxygenation seems to be available to patients who are waiting for an impending lung transplant [11]. These only detail the supportive care and not the medical advances described by RAGHU [1], which are, in our opinion, fundamental.

As briefly discussed, research continues in the pharmacological management of IPF-related AEs [1]. It still typically includes high-dose corticosteroids and/or immunosuppressant and broad-spectrum antibiotics, despite the scarcity of data supporting the usefulness of these therapies [2]. A placebo-controlled randomised phase III trial evaluating the efficacy of cyclophosphamide in conjunction with corticosteroids in IPF-related AEs was recently approved in France (EXAFIP; registered at ClinicalTrials.gov with the identifier number NCT02460588). A number of other novel therapeutic approaches (nintedanib, pirfenidone, polymyxin B direct haemoperfusion, thrombomodulin, rituximab, plasma exchange, intravenous immunoglobulin) have

@ERSpublications

IPF-related acute exacerbations: further research needed http://ow.ly/CB3W30hzYNq

Cite this article as: Vincent F, Nunes H, Gaudry S. Progress in the management of IPF-related acute exacerbations: a goal for patients, respirologists and intensivists. Eur Respir J 2018; 51: 1702419 [https:// doi.org/10.1183/13993003.02419-2017]. 
been evaluated or are still being evaluated; however, preliminary results are disappointing or are based on too small series [2]. We believe that RAGHU [1] emphasises above all the lack of relevant data on the pharmacological management of IPF-related AEs and the need for further studies.

François Vincent ${ }^{1}$, Hilario Nunes ${ }^{2,3}$ and Stéphane Gaudry ${ }^{4,5}$

${ }^{1}$ Réanimation polyvalente, Groupe Hospitalier Intercommunal Le-Raincy Montfermeil, Montfermeil, France. ${ }^{2}$ Service de Pneumologie, Hôpital Avicenne, Assistance Publique - Hôpitaux de Paris, Bobigny, France. ${ }^{3}$ Université Paris 13, Sorbonne Paris Cité, EA2363 'Hypoxie et poumon', Paris, France. ${ }^{4}$ Service de Réanimation Médico-Chirurgicale, Hôpital Louis Mourier, Assistance Publique - Hôpitaux de Paris, Colombes, France. ${ }^{5}$ Epidémiologie Clinique et Évaluation Économique Appliqué aux Populations Vulnérables (ECEVE), UMR 1123, Université Paris Diderot, Sorbonne Paris Cité, Paris, France.

Correspondence: François Vincent, Réanimation polyvalente, Groupe Hospitalier Intercommunal Le-Raincy Montfermeil, 10 rue du Général Leclerc, 93370, Montfermeil, France. E-mail: frncsvncnt@aol.com

Received: Nov 232017 | Accepted after revision: Nov 292017

Conflict of interest: None declared.

\section{References}

1 Raghu G. Idiopathic pulmonary fibrosis: lessons from clinical trials over the past 25 years. Eur Respir J 2017; 50: 1701209 .

2 Kondoh Y, Cottin V, Brown KK. Recent lessons learned in the management of acute exacerbation of idiopathic pulmonary fibrosis. Eur Respir Rev 2017; 26: 170050.

3 Martinez FJ, Collard HR, Pardo A, et al. Idiopathic pulmonary fibrosis. Nat Rev Dis Primers 2017; 3: 17074.

4 Spagnolo P, Wuyts W. Acute exacerbations of interstitial lung disease: lessons from idiopathic pulmonary fibrosis. Curr Opin Pulm Med 2017; 23: 411-417.

5 Raghu G, Collard HR, Egan JJ, et al. An official ATS/ERS/JRS/ALAT statement: idiopathic pulmonary fibrosis: evidence-based guidelines for diagnosis and management. Am J Respir Crit Care Med 2011; 183: 788-824.

6 Raghu GG, Rochwerg BB, Zhang YY, et al. An official ATS/ERS/JRS/ALAT clinical practice guidelines: treatment of idiopathic pulmonary fibrosis. An update of the 2011 Clinical Practice Guideline. Am J Respir Crit Care Med 2015; 192: e3-e19.

7 Cottin V, Crestani B, Cadranel J, et al. French practical guidelines for the diagnosis and management of idiopathic pulmonary fibrosis - 2017 update. Full-length version. Rev Mal Respir 2017; 34: 900-968.

8 Kang HS, Cho KW, Kwon SS, et al. Prognostic significance of Glasgow prognostic score in patients with acute exacerbation of idiopathic pulmonary fibrosis. Respirology 2018; 23: 206-212.

9 Boyer A, Vargas F, Delacre M, et al. Prognostic impact of high-flow nasal cannula oxygen supply in an ICU patient with pulmonary fibrosis complicated by acute respiratory failure. Intensive Care Med 2011; 37: 558-559.

10 Vianello A, Arcaro G, Battistella L, et al. Noninvasive ventilation in the event of acute respiratory failure in patients with idiopathic pulmonary fibrosis. J Crit Care 2014; 29: 562-567.

11 Trudzinski FC, Kaestner F, Schafers HJ, et al. Outcome of patients with interstitial lung disease treated with extracorporeal membrane oxygenation for acute respiratory failure. Am J Respir Crit Care Med 2016; 193: 527-533.

Copyright (CERS 2018

From the author:

I appreciate the expressed views of F. Vincent and colleagues, regarding the need to address treatment consideration of acute exacerbation (AE) of idiopathic pulmonary fibrosis (IPF). While the mortality of patients manifesting AE of IPF is unfortunately high, F. Vincent and colleagues argue that supportive care in the intensive care unit (ICU) is an appropriate consideration, including noninvasive and/or mechanical ventilation for patients. Indeed, well-informed patients may opt for such aggressive supportive measures in the ICU, and for some patients listed for lung transplantation, such aggressive supportive measures may serve as a "bridge" for a suitable lung to become available during a set time. Such decisions will need to be made on a case by case basis and tailored to patients' preferred choices, advance directives that patients may have in place and other individual needs. In addition, other confounding factors, such as comorbid conditions if present, which may have a poor outcome otherwise, should be taken into consideration when providing aggressive supportive care in the ICU for patients manifesting AE of IPF.

@ERSpublications

Until clinical trial results are available, treatment decisions for acute exacerbations of IPF must be made on an individual basis http://ow.ly/FFxi30hY7uz

Cite this article as: Raghu G. Progress in the management of IPF-related acute exacerbations: a goal for patients, respirologists and intensivists. Eur Respir J 2018; 51: 1800113 [https://doi.org/10.1183/ 13993003.00113-2018]. 
The need for clinical trials in this subgroup of patients manifesting AE of IPF is evident. Although AzUmA et al. [1] reported $\sim 15 \%$ of patients in the placebo arm to have manifested AE of IPF in a phase II clinical trial, all other clinical trials to date have reported much lower percentages of patients manifesting AE of IPF [2]. The difficulty in obtaining all the necessary clinical data with a high-resolution computed tomography chest scan to meet the criteria for AE of IPF, leading to missing data from centres unable to perform all the required evaluation to ascertain the diagnosis of AE of IPF, results in additional difficulties in designing the much needed well-designed clinical trials [3]. The need for adjudication of the events as true AE of IPF is imperative. Given the relatively small percentage of patients manifesting AE of IPF in clinical trials to date [2], the feasibility of enrolling the required sample size to determine the efficacy of a treatment regimen will be challenging and will require large numbers of patients recruited from several specialised centres worldwide.

Acknowledging these factors, indeed, well-designed clinical trials to determine a safe and efficacious pharmacotherapeutic intervention for the AE of IPF are much needed. In this regard, it is with great enthusiasm that we look forward to successful outcomes for patients with IPF manifesting AE of IPF in the recently approved placebo-controlled randomised phase III trial evaluating the efficacy of cyclophosphamide in conjunction with corticosteroids in IPF-related AEs in France (EXAFIP; ClinicalTrials.gov identifier NCT02460588). The results of this clinical trial are eagerly waited.

Ganesh Raghu

Center for Interstitial Lung Diseases, University of Washington, Seattle, WA USA.

Correspondence: Ganesh Raghu, Center for Interstitial Lung Diseases, University of Washington, Seattle, WA 98195, USA. E-mail: graghu@uw.edu

Received: Jan 182018 | Accepted: Jan 182018

Conflict of interest: G. Raghu reports personal fees from Boehringer-Ingelheim, Sanofi and Veracyte; and other fees as a consultant for IPF studies from Boehringer-Ingelheim, BMS, Fibrogen, Gilead, Promedior, Roche-Genentech, Sanofi, United Therapeutics, UCB and Veracyte; all outside the submitted work.

\section{References}

1 Azuma A, Nukiwa T, Tsuboi E, et al. Double-blind, placebo-controlled trial of pirfenidone in patients with idiopathic pulmonary fibrosis. Am J Respir Crit Care Med 2005; 171: 1040-1047.

2 Raghu G. Idiopathic pulmonary fibrosis: lessons from clinical trials over the past 25 years. Eur Respir J 2017; 50: 1701209.

3 Collard HR, Ryerson CJ, Corte TJ, et al. Acute exacerbation of idiopathic pulmonary fibrosis. An international working group report. Am J Respir Crit Care Med 2016; 194: 265-275. 